\title{
Blocking the CCL5-CCR5 Axis Using Maraviroc Promotes MI Polarization of Macrophages Cocultured with Irradiated Hepatoma Cells
}

\author{
Yuan Zhuang* \\ Xiaomei Zhao* \\ Baoying Yuan \\ Zhaochong Zeng (D) \\ Yixing Chen (D)
}

Department of Radiation Oncology, Zhongshan Hospital, Fudan University, Shanghai, People's Republic of China

*These authors contributed equally to this work
Correspondence: Yixing Chen;

Zhaochong Zeng

Department of Radiation Oncology,

Zhongshan Hospital, Fudan University,

I80 Fenglin Road, Shanghai, 200032,

People's Republic of China

Tel +86-139-1605-6575

Fax +86-2I-6404-8472

Email chen.yixing@zs-hospital.sh.cn;

zeng.zhaochong@zs-hospital.sh.cn
Purpose: The C-C chemokine ligand 5 (CCL5)-C-C chemokine receptor (CCR5) axis facilitates tumor progression via multiple mechanisms. Herein, we elucidated the effect of a CCR5 antagonist (maraviroc [MVC]; blocking the CCL5-CCR5 axis) on the phenotype of macrophages cocultured with irradiated hepatoma cells. In addition, we investigated whether modulation of macrophage polarization can alter tumor cell sensitivity to radiation.

Materials and Methods: Quantitative reverse-transcription polymerase chain reaction, Western blotting, and enzyme-linked immunosorbent assays were applied to examine the levels of macrophage-associated markers. The mechanisms of macrophage polarization were explored by Western blotting in an in vitro model of coculture of human hepatoma cells with macrophages. The radiation sensitivity was examined in a clonogenic radiosensitivity assay. Tumor cell apoptosis was detected by Western blotting and flow cytometry. A mouse model of a subcutaneous tumor was also established.

Results: CCL5 skewed THP-1 M0 macrophages toward an M2-like phenotype. In coculture with hepatoma cells, macrophages manifested high levels of interleukin (IL) 10, IL-12, tumor necrosis factor $\alpha(\mathrm{TNF}-\alpha)$, transforming growth factor $\beta 1$ (TGF- $\beta 1)$, arginase 1 (ARG1), and IL-1 $\beta$. Tumor cell irradiation further upregulated these markers in macrophages. After incubation of macrophages with MVC for $24 \mathrm{~h}$, levels of M1 cytokines significantly increased, whereas those of M2 phenotype factors ARG1, TGF- $\beta 1$, and IL-10 decreased, accompanied by the activation of signal transducer and activator of transcription 3 (STAT3) and downregulation of suppressor of cytokine signaling 3 (SOCS3). The macrophage phenotype reverted to M2 states after treatment with a STAT3 inhibitor. The shift of macrophages toward the M1 phenotype enhanced the radiosensitivity and apoptosis of hepatoma cells. Mice receiving a combination of X-ray irradiation and MVC experienced a better antitumor effect than those receiving either MVC or irradiation alone did.

Conclusion: M2 polarization of macrophages induced by CCL5-CCR5 signaling can be inhibited using MVC via the STAT3-SOCS3 pathway. The shift of macrophages toward the M1 phenotype promotes the sensitivity of human hepatoma cells to X-ray irradiation.

Keywords: hepatocellular carcinoma, CCL5, CCR5, macrophage, polarization, maraviroc

\section{Introduction}

Radiation therapy is a safe and effective method for the management of hepatocellular carcinoma (HCC). ${ }^{1}$ Nonetheless, the recurrence outside a radiation field and radiation therapy-associated tumor progression are still the main causes of treatment failure. ${ }^{2}$ Some studies ${ }^{2-4}$ have revealed that a microenvironment change after irradiation is an 
important reason for these failures, where macrophages play a vital role. Macrophages are highly plastic cells that can be broadly categorized into the antitumoral M1 phenotype and the protumoral M2 phenotype, ${ }^{5}$ and tumor-associated macrophages (TAMs) have been shown to have an M2-like phenotype. ${ }^{6}$ Moreover, postirradiation tumors carry out greater recruitment of TAMs, ${ }^{7-9}$ which are strongly associated with tumor progression and metastasis. ${ }^{10-12}$ Based on the above theories, switching TAMs to a predominantly M1-like phenotype has been proposed as a promising immunotherapeutic treatment strategy. ${ }^{13,14}$

In the initiation and progression of $\mathrm{HCC}$, the interaction of chemokines with their receptors influences these processes by promoting inflammation, angiogenesis, and metastasis. $^{15,16} \mathrm{C}-\mathrm{C}$ chemokine ligand 5 (CCL5, also known as RANTES), a member of the CC family of chemokines whose expression and secretion are regulated in immune cells and tumor cells, ${ }^{17}$ is receiving increasing attention. Although CCL5 can bind to receptors C-C chemokine receptor (CCR) 1 and CCR3, the biological activity of CCL5 is mainly mediated by the binding to CCR5. ${ }^{18}$ CCR5 is the main coreceptor for HIV-1 cell entry and binds with a high affinity to CCL5, CCL3, and CCL4. ${ }^{19,20}$ Numerous preclinical studies have confirmed a key role of the CCL5-CCR5 axis in the recruitment of TAMs to a tumor site and in their reprogramming into immunosuppressive cells in various cancers. $^{18,21,22}$ As for HCC, the important function of CCL5 and/or CCR5 in the initiation and progression of liver cancer has been determined. ${ }^{23-25}$ Nonetheless, the CCL5-CCR5 axis-mediated crosstalk between TAMs and HCC cells and a possible treatment targeting this axis are unclear.

Growing evidence suggests potential strategies for decreasing CCL5 secretion $^{26-28}$ or for inhibiting the CCR5-CCL5 signaling pathway ${ }^{21,29-33}$ in different types of cancers. The present study is focused on maraviroc (MVC), a highly selective and FDA-approved CCR5 antagonist that was originally developed for patients with $\mathrm{HIV}^{34}$ Of note, we found that MVC skews the phenotype of macrophages exposed to irradiated hepatoma cells toward an antitumor M1 state via the signal transducer and activator of transcription 3 (STAT3)-suppressor of cytokine signaling 3 (SOCS3) pathway. We also demonstrated that MVC indirectly enhances radiation sensitivity of hepatoma cells in vitro and impedes subcutaneous growth of liver tumor cells in mice, thereby offering a promising therapeutic strategy against HCC.

\section{Materials and Methods Cell Lines and Cell Culture}

Two human hepatoma cell lines (HCCLM3 and Huh7) were obtained from the Liver Cancer Institute of Fudan University (Shanghai, China). Human monocytic THP-1 cells were purchased from the Institute of Biochemistry and Cell Biology, the Chinese Academy of Sciences (Shanghai, China). The Ethics Committee of Zhongshan Hospital, Fudan University, approved the experimental work involving cell lines. All cell lines were maintained in the Roswell Park Memorial Institute (RPMI) 1640 medium (KeyGen Biotechnology) supplemented with $10 \%$ of fetal bovine serum (Gibco BRL). THP-1 monocytes were differentiated into THP-1 M0 macrophages by $48 \mathrm{~h}$ incubation with $100 \mathrm{ng} /$ $\mathrm{mL}$ phorbol 12-myristate 13-acetate (PMA, Sigma) followed by $24 \mathrm{~h}$ incubation in the RPMI 1640 medium. THP-1 M0 macrophages were next polarized into M1 macrophages by treatment with $100 \mathrm{ng} / \mathrm{mL}$ lipopolysaccharide (R\&D Systems) and $20 \mathrm{ng} / \mathrm{mL}$ interferon $\gamma$ (R\&D Systems) for 24 h. To generate M2-polarized macrophages, M0 macrophages were incubated with $20 \mathrm{ng} / \mathrm{mL}$ interleukin (IL) 4 (R\&D Systems) and IL-13 (R\&D Systems) for $24 \mathrm{~h}$.

\section{Hepatoma Cell Irradiation}

HCCLM3 and Huh7 hepatoma cells were irradiated on an $\mathrm{ONCOR}^{\mathrm{TM}}$ linear accelerator (Siemens, Munich, Germany) at a dose rate of $300 \mathrm{cGy} / \mathrm{min}$. The irradiation field ranged from $15 \times 15$ to $40 \times 40 \mathrm{~cm}$ according to the number of cell plates. Hepatoma cell lines received a single 8 Gy dose of X-ray irradiation with an energy of $6 \mathrm{MV}$.

\section{Coculture of Human Hepatoma Cells with THP-I Macrophages}

Human THP-1 cells were seeded in each well of a 6-well upper Transwell insert $(0.4 \mu \mathrm{m}$ microporous membrane, Corning) and placed in a separate 6-well plate containing PMA for polarization. On the day before macrophage polarization was completed, HCCLM3 and Huh7 cells were seeded in fresh 6-well lower Transwell plates and were allowed to attach overnight. After the polarization was completed, THP-1 macrophages in the upper Transwell inserts were carefully washed with phosphatebuffered saline (KeyGen Biotechnology), and each upper Transwell insert was transferred to a 6-well lower Transwell plate containing hepatoma cells. The polarized macrophages and hepatoma cells were then cocultured in the RPMI 1640 medium for $24 \mathrm{~h}$. 


\section{Quantitative Reverse-Transcription Polymerase Chain Reaction (qRT-PCR)}

Total RNA was extracted from cell lines using the TRIzolTM Reagent (Invitrogen) and was reversetranscribed into single-stranded complementary DNA using the PrimeScript ${ }^{\mathrm{TM}}$ RT Reagent Kit (Takara Bio Inc.). Subsequent quantitative PCR amplification was performed with $\mathrm{SYBR}^{\mathrm{TM}}$ Premix Ex Taq ${ }^{\mathrm{TM}}$ (TaKaRa Bio Inc.) on an Applied Biosystems 7500 real-time detection system (Applied Biosystems, Carlsbad, CA, USA). Primers for IL-12, IL-10, tumor necrosis factor $\alpha$ (TNF$\alpha$ ), transforming growth factor $\beta 1$ (TGF- $\beta 1$ ), CCL5, and CCR5 cDNAs were designed by Sangon Biotech and are listed in Table S1. $\beta$-Actin served as an internal standard control for messenger RNA (mRNA) detection. Gene expression was quantified by the $2^{-\Delta \Delta \mathrm{Ct}}$ method.

\section{Cell Transfection}

Small interfering RNAs (siRNAs) specific for CCL5 and SOCS3 mRNA and their negative controls were purchased from Ribobio (Guangzhou, China), and the sequences are listed in Table S2. Five nanomoles of siRNA was dissolved in $250 \mu \mathrm{L}$ of RNase-free $\mathrm{H}_{2} \mathrm{O}$ to prepare a $20 \mu \mathrm{M}$ stock solution. Transfection reagent INTERFERin ${ }^{\circledR}$ was added to the siRNA solution, vortex-mixed, and incubated for $10 \mathrm{~min}$ at room temperature. INTERFERin ${ }^{\circledR}-$ siRNA complexes were added to each plate by pipetting the mixture up and down. Finally, the cells were incubated at $37{ }^{\circ} \mathrm{C}$ for $24 \mathrm{~h}$ to collect RNA and for $48 \mathrm{~h}$ to extract total protein.

\section{Clonogenic Radiosensitivity Assay}

M1 or M2 macrophages were cocultured with HCCLM3 or Huh7 hepatoma cells for $24 \mathrm{~h}$ in a Transwell system as described above. Hepatoma cells were then seeded in 6-well plates without macrophages and allowed to attach for $6 \mathrm{~h}$, followed by different doses of X-ray radiation $(0$, 2, 4, 6, and $8 \mathrm{~Gy}$ ). After the irradiation, the hepatoma cells were cultured for additional 2 weeks and then subjected to Giemsa staining to calculate the remaining adherent colonies of no less than 50 cells. After that, colony-forming efficiency was determined. The multitarget click model surviving fraction " $1-\left(1-\mathrm{e}^{-\mathrm{D} / \mathrm{D} 0}\right) \mathrm{N}$ " was employed to delineate the survival curve in GraphPad Prism software (version 8.0).

\section{Western Blot Analysis}

Cells were lysed with radioimmunoprecipitation assay buffer in the presence of protease and phosphatase inhibitor cocktails (Beyotime, China). Total protein concentration was determined by means of the Bicinchoninic Acid Assay Kit (Beyotime, China). Equal amounts of the protein extracts were loaded onto a polyacrylamide gel for SDS-PAGE, and afterwards, the proteins were transferred onto polyvinylidene difluoride membranes (Millipore). The membranes were probed with primary antibodies at $4{ }^{\circ} \mathrm{C}$ overnight, followed by incubation with horseradish peroxidase-conjugated secondary antibodies for $1 \mathrm{~h}$ at room temperature. Antibodies against SOCS3 (cat. \# ab16030), Janus kinase 2 (JAK2, ab108596), phosphorylated JAK2 (ab32101), STAT3 (ab68153), CCAAT/enhancer-binding protein (cEBP/ $\beta$, ab32358), phosphorylated $\mathrm{cEBP} / \beta$ (ab52194), phosphorylated extracellular regulated protein kinases 1/2 (ERK1/2, ab76299), p38 mitogenactivated protein kinase (MAPK, ab170099), phosphorylated p38 MAPK (ab195049), IL-1 $\beta$ (ab156791), arginase 1 (Arg-1, ab239731), and CCL5 (ab9679) were bought from Abcam. Antibodies against beta-tubulin (cat. \# 2146) and phosphorylated STAT3 (\#9145) were purchased from Cell Signaling Technology Inc. (Danvers, MA, USA), whereas anti-ERK1/2 (ARG65350) and antiCCR5 (ARG59254) antibodies from Arigo biolaboratories (Hsinchu City, Taiwan, China). The blots were visualized using a chemiluminescence system (Bio-Rad, Hercules, CA, USA). The intensity of each protein band was quantified in Quantity One 4.6.2 software (Bio-Rad).

\section{Enzyme-Linked Immunosorbent Assays (ELISAs)}

Concentrations of CCL5, IL-12, IL-10, TNF- $\alpha$, and TGF$\beta 1$ secreted by THP-1 macrophages were measured by means of ELISA kits (R\&D Systems). Three biological replicates were analyzed. Absorbance was recorded at 450 $\mathrm{nm}$ on a microplate reader.

\section{Flow Cytometry (FCM)}

HCCLM3 and Huh7 cells in the exponential growth phase were cultured in 6-well plates for $24 \mathrm{~h}$. The hepatoma cells were harvested, stained with Annexin- $\mathrm{V}$ and propidium iodide (PI; BD Biosciences, San Jose, CA), and subjected to FCM. 


\section{Animal Experiments}

Male C57BL/6J mice (5 weeks old) were obtained from the Shanghai SLAC Laboratory Animal Co., Ltd. Luciferasetagged $\mathrm{H} 22$ liver tumor cells $\left(5 \times 10^{6}\right)$ in $100 \mu \mathrm{L}$ of phosphatebuffered saline were injected into the left thigh to create a subcutaneous tumor. When the tumor grew to $\sim 1 \mathrm{~cm}$ size, the mice were randomized into four groups: a negative control group, X-ray irradiation group, MVC administration group, and irradiation combined with MVC group. MVC was purchased from Selleck Chemicals and injected intraperitoneally for 3 consecutive weeks (10 $\mathrm{mg} / \mathrm{kg}$, daily). For the X-ray treatment, the mice were laid on the right side after abdominal anesthesia. X-ray irradiation (16 Gy in 1 fraction) was administered to subcutaneous tumors with an energy of $6 \mathrm{MV}$. The research protocol was approved by the Animal Ethics Committee of Zhongshan Hospital, Fudan University, in compliance with the China Guideline for ethical review of animal welfare (GB/T 35892-2018).

\section{Statistical Analyses}

SPSS 23.0 statistical software (version 23.0, SPSS Inc.) was utilized for data analysis. Continuous variables were analyzed by the independent $t$-test or nonparametric Mann-Whitney $U$-test. Tumor growth curves were compared by a two-way analysis of variance. Statistical significance was set to $P<0.05$.

\section{Results}

\section{CCL5 Polarizes THP-I Macrophages Toward an M2-Like Phenotype}

First, we confirmed that THP-1 M0 macrophages differentiated with PMA can secrete CCL5 (Figure 1A). Then, we knocked down CCL5 (Figure 1B) and examined mRNA levels of known cytokines expressed by macrophages in the M1 (IL-12 and TNF- $\alpha$ ) and M2 (TGF- $\beta 1$ and IL-10) states. Our results suggested enhanced expression of IL-12 and decreased expression of IL-10 in the si-CCL5 group compared to that in the control group $(P<0.05$, Figure $1 \mathrm{C})$. Nevertheless, there were no significant differences in the expression of TGF- $\beta 1$ and TNF- $\alpha$. To further verify the role of CCL5 in the modulation of macrophage polarization, THP-1 macrophages were treated with various concentrations of CCL5 (0-20 ng/mL) for $24 \mathrm{~h}$. As depicted in Figure 1D, low concentrations $(5-10 \mathrm{ng} / \mathrm{mL})$ of CCL5 typically skewed M0 macrophages toward an M2-like phenotype, which was characterized by decreased mRNA levels of TNF- $\alpha$ and IL-12 and increased levels of
IL-10 and TGF- $\beta 1$. However, when the concentration of CCL5 reached $20 \mathrm{ng} / \mathrm{mL}$, the expression levels of all these cytokine genes were increased (Figure 1D). Notably, the upregulation of M2 marker TGF- $\beta 1$ was the highest ( $>5$-fold) as compared to that of TNF- $\alpha$, IL-12, and IL10 (1- to 2-fold). Similarly, the above trends were observed at the protein level using ELISA (Figure 1E). Taken together, these results suggested that macrophages responded differently to various concentrations of CCL5, which can modify their functional phenotype.

\section{CCR5 Antagonist Reprograms the Polarization of Macrophages Cocultured with Irradiated Hepatoma Cells into the MI State by Blocking the CCL5-CCR5 \\ Axis}

To test whether the CCR5 antagonist MVC can modulate macrophage plasticity by blocking the CCL5-CCR5 axis, we added CCL5 (5 ng/mL) and/or $5 \mu \mathrm{M}$ MVC (Figure S1) into the macrophage culture medium and analyzed changes in the expression of macrophage markers in comparison with those in the control. As presented in Figure 2A and B, MVC significantly decreased the expression level of CCR5 and M2 polarization of THP-1 macrophages induced by CCL5. To identify the effects of hepatoma cells on macrophages, we constructed a coculture system of THP-1 M0 macrophages and HCCLM3 or Huh7 hepatoma cells (with or without $\mathrm{X}$-ray irradiation). Of note, our data uncovered significantly higher levels of cytokines, including TNF- $\alpha$, TGF- $\beta 1$, IL-10, and IL-12, in macrophages exposed to tumor cells in comparison with the levels in the control (Figure 2C). The expression levels of these markers in macrophages further increased when the tumor cells were exposed to 8 Gy X-ray irradiation $(P<0.05)$. Notably, macrophages incubated with MVC for 24 $\mathrm{h}$ featured decreased mRNA expression levels of IL-10 and TGF- $\beta 1$, accompanied by the upregulation of TNF- $\alpha$ and IL-12, relative to macrophages without MVC treatment $(P<0.05$, Figure 2C). Furthermore, the results of Western blotting and ELISAs confirmed the ability of MVC to shift macrophages toward the M1 phenotype at the protein level, as evidenced by the upregulation of IL-1 $\beta$ and IL-12 and the downregulation of IL-10 and arginase 1 (ARG1, a marker of M2 macrophages) (Figure 2D and E). 
A

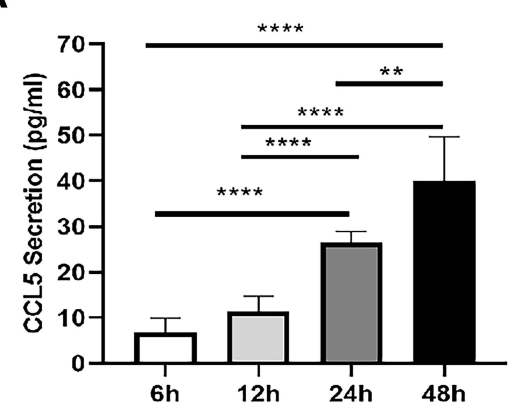

B

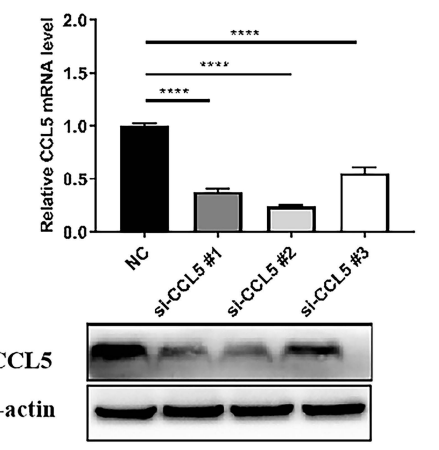

C

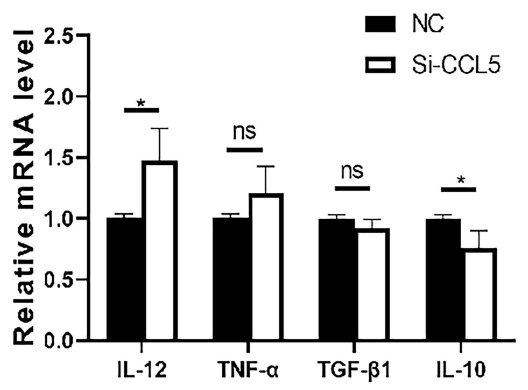

D

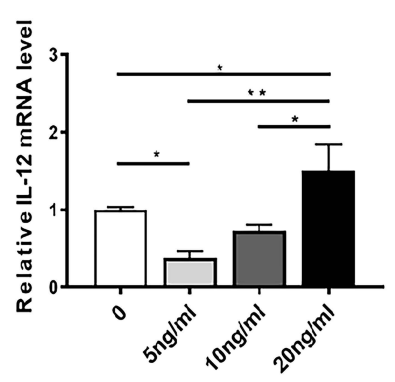

$\mathbf{E}$

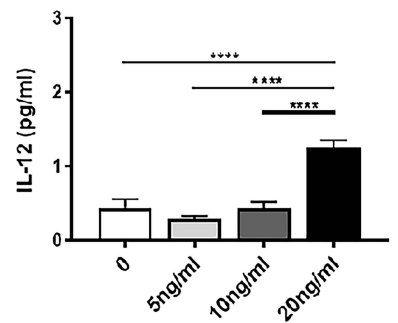

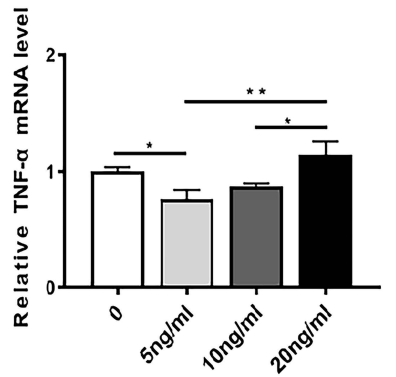
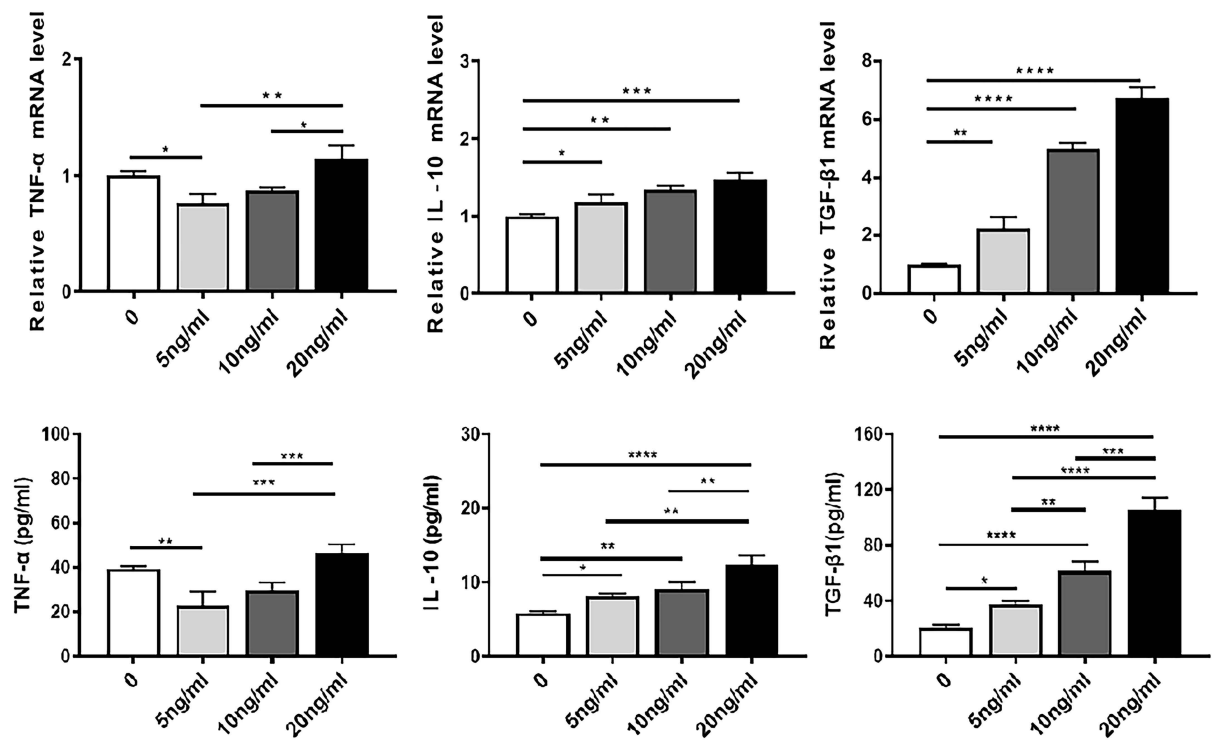

Figure I CCL5 polarizes THP-I M0 macrophages toward an M2-like phenotype. Human THP-I monocytes were differentiated into THP-I M0 macrophages by incubation with PMA ( $100 \mathrm{ng} / \mathrm{mL}$ ) for $48 \mathrm{~h}$. (A) An ELISA of CCL5 in the supernatant of THP-I M0 macrophages cultured for $6-48 \mathrm{~h}$. (B and C) The most efficient siRNA against CCL5 (labeled as si-CCL5) \#2 was determined by QRT-PCR and Western blotting (B) and then transfected into THP-I M0 macrophages. Expression levels of markers of the MI phenotype (IL12 and TNF- $\alpha$ ) and M2 phenotype (IL- 10 and TGF- $\beta$ I) were measured by qRT-PCR in transfected THP-I M0 macrophages (C). (D) qRT-PCR analysis of the expression of macrophage-associated cytokines including IL-I2, TNF- $\alpha$, IL-I0, and TGF- $\beta$ I in THP-I M0 macrophages treated with CCL5 at different concentrations (0, 5, I0, or 20 ng/mL) for 24 h. (E) ELISAs of IL- I2, TNF- $\alpha$, IL- I0, and TGF- $\beta$ I in the culture supernatant of THP-I M0 macrophages treated with CCL5 at different concentrations (0, 5 , I0, or 20 ng/mL) for $24 \mathrm{~h}$. Values represent mean \pm standard deviation from three independent experiments. $* P<0.05 ; * * P<0.01 ; * * * P<0.00$ I; ****P< 0.000 I.

Abbreviations: NC, negative control; ns, no significance.

\section{The CCL5-CCR5 Axis Regulates the Macrophage Phenotype Through the STAT3-SOCS3 Pathway}

To identify the pathway involved in the alteration of macrophage polarization, Western blotting was carried out to assess the expression of the SOCS3 protein and total protein levels and phosphorylated-protein levels of p38 MAPK, ERK1/2, JAK2, STAT3, and cEBP/ $\beta$, which represent the main pathways linked with CCL5CCR5 signal transduction. As shown in Figure 3A, with increasing concentrations of CCL5, SOCS3 protein expression markedly increased, while the levels of total and phosphorylated STAT3 significantly diminished. To identify the involvement of SOCS3 in the modulation of macrophage polarization, we knocked down SOCS3 by the siRNA technique (Figure 3B and $\mathrm{C}$ ) and noticed that the mRNA expression of M1 marker IL-12 increased, while the expression of M2 marker IL-10 declined (Figure 3D). The results revealed that the SOCS3 deficiency skewed macrophages toward an M1-like phenotype. Next, to verify whether MVC can reverse the downregulation of the STAT3 protein induced by CCL5, THP-1 M0 macrophages were treated with MVC for $24 \mathrm{~h}$, and the expression of proteins of the JAK2-STAT3 signaling pathway and of its feedback inhibitor SOCS3 was evaluated by Western blotting. As depicted in Figure 3E, MVC activated the phosphorylation of STAT3 and attenuated the upregulation of SOCS3 induced by 
A
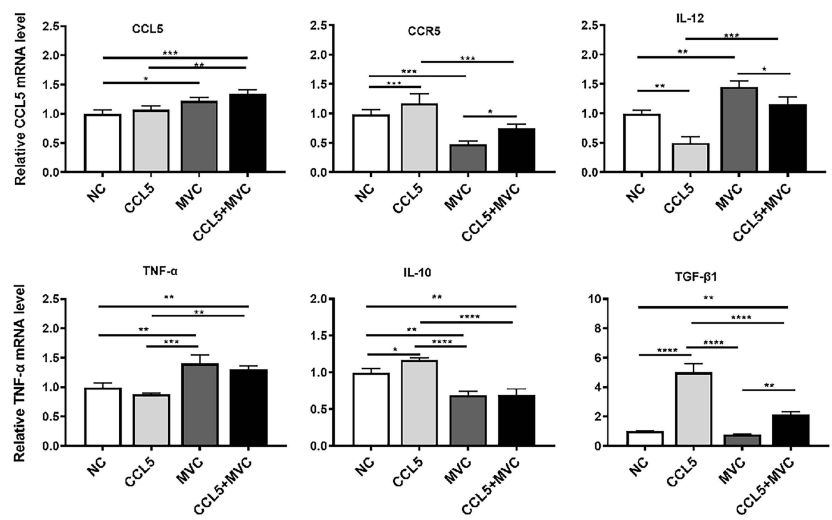

B
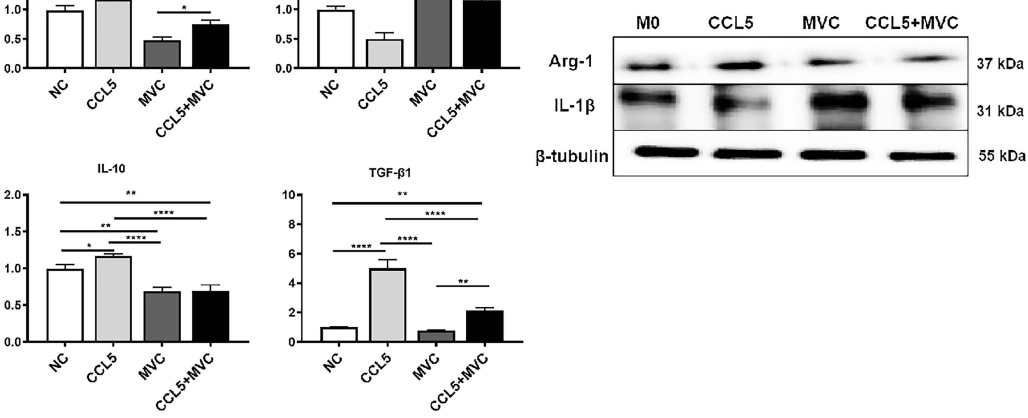

C

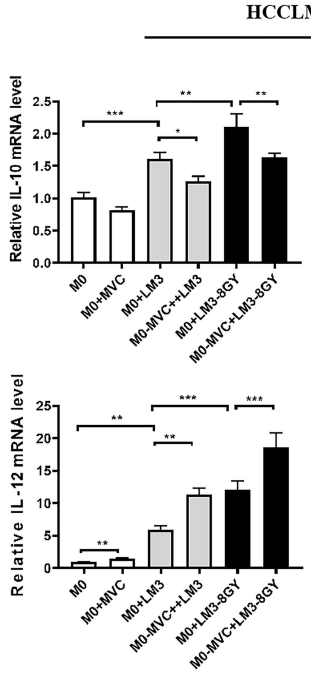

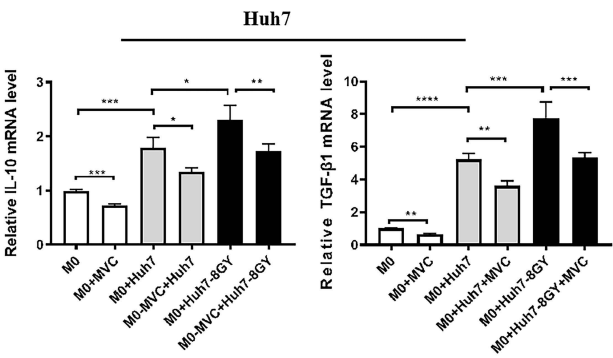
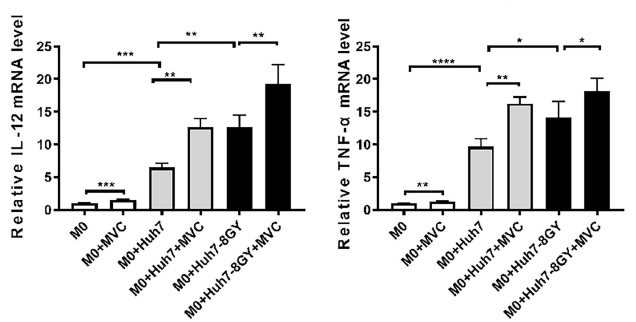

Huh7
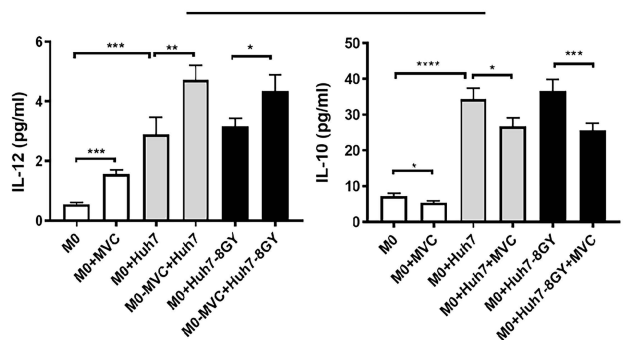

E
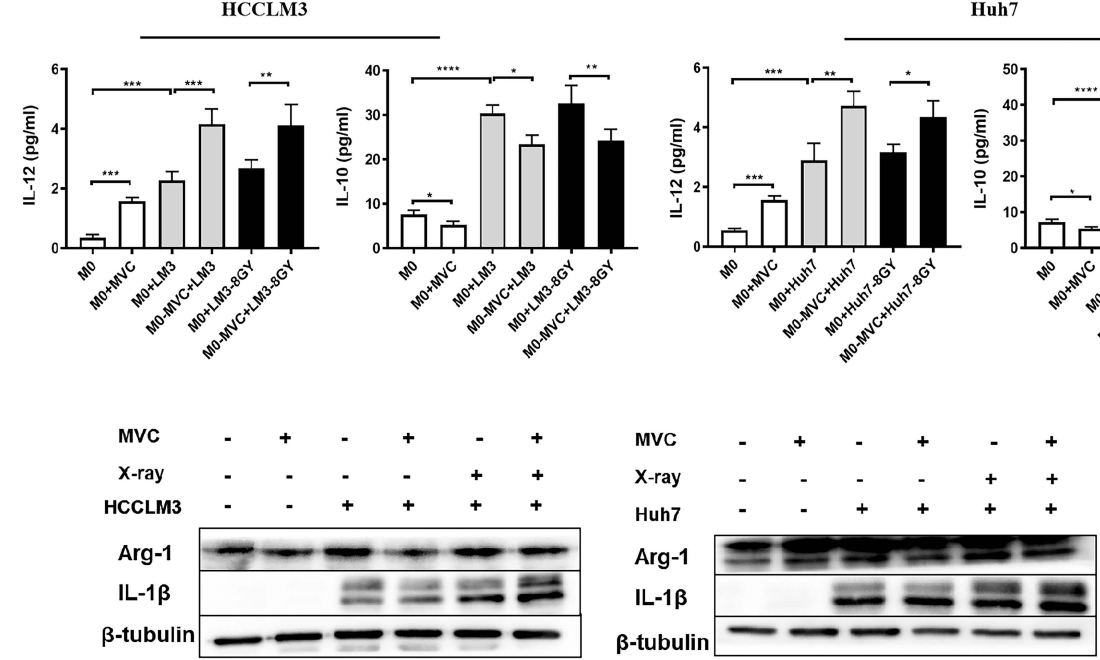

Figure 2 MVC reprograms the polarization of macrophages cocultured with hepatoma cells toward an MI-like phenotype. (A) qRT-PCR analysis of the expression of CCL5, CCR5, TNF- $\alpha$, IL-12, TGF- $\beta$ I, and IL- 10 in THP-I M0 macrophages incubated with CCL5 (5 ng/mL) and/or MVC (5 $\mu \mathrm{M})$ for 24 h. (B) Representative Western blots showing the expression of ARGI (a marker of M2 macrophages) and IL-I $\beta$ (a marker of MI macrophages) in THP-I M0 macrophages incubated with CCL5 (5 ng/mL) and/or MVC (5 $\mu \mathrm{M})$ for $24 \mathrm{~h}$. (C) qRT-PCR analysis of the expression of TNF- $\alpha$, IL-I2, TGF- $\beta$ I, and IL-10 in THP-I M0 macrophages cocultured with either HCCLM3 or Huh7 hepatoma cells with or without 8 Gy X-ray irradiation. (D) ELISAs of IL-I2 and IL-10 in the culture supernatant of THP-I M0 macrophages cocultured with either HCCLM3 or Huh7 hepatoma cells subjected or not subjected to 8 Gy X-ray irradiation. (E) Representative Western blots illustrating the expression of ARGI and IL-I $\beta$ in THP-I M0 macrophages cocultured with hepatoma cells subjected or not subjected to $8 \mathrm{~Gy}$ X-ray irradiation. The data represent mean \pm standard deviation from three independent experiments. $* P<0.05 ; * * P<0.01 ; * * * P<0.001 ; * * * * P<0.0001$.

Abbreviations: NC, negative control; MVC, maraviroc. 


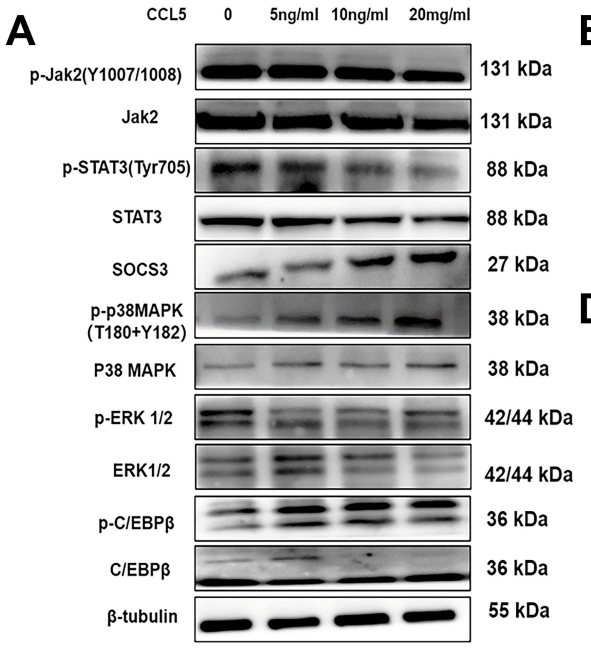

F
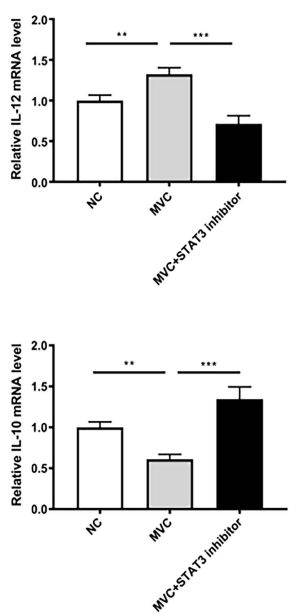

B

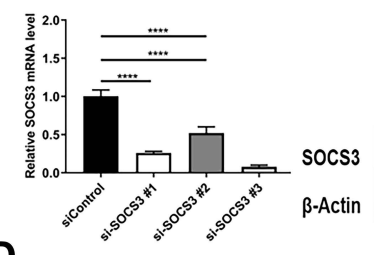

E

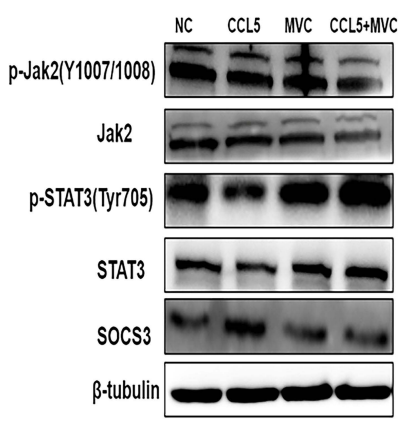

C

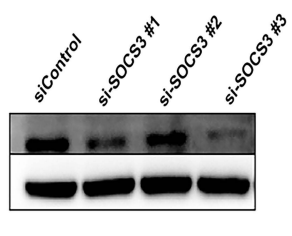

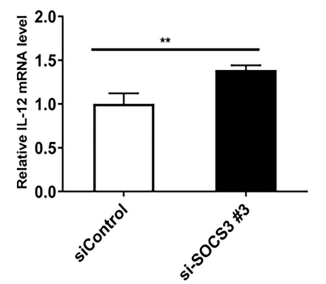

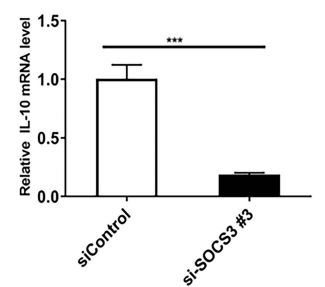

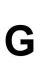

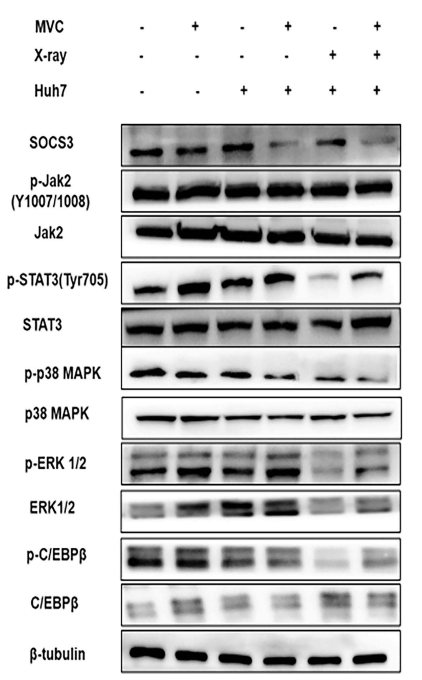

Figure 3 The CCL5-CCR5 axis modulates macrophage polarization through the STAT3-SOCS3 pathway. (A) Representative Western blots showing the expression of the SOCS3 protein as well as total protein levels and phosphorylated-protein levels of JAK2, STAT3, P38 MAPK, ERKI/2, and cEBP/ $\beta$ in THP-I M0 macrophages treated with CCL5 $(0,5,10$, or $20 \mathrm{ng} / \mathrm{mL})$ for $24 \mathrm{~h}$. (B-D) The most efficient siRNA against SOCS3 (labeled as si-SOCS3) \#3 was determined by qRT-PCR (B) and Western blotting (C) and then transfected into THP-I M0 macrophages. The markers of MI (IL-I2) and M2 (IL-I0) macrophages were quantitated by qRT-PCR in transfected THP-I M0 macrophages (D). (E) Representative Western blots revealing the expression of the SOCS3 protein as well as total protein levels and phosphorylated-protein levels of JAK2 and STAT3 in THP-I M0 macrophages treated with CCL5 $(5 \mathrm{ng} / \mathrm{mL})$ and/or MVC (5 $\mu \mathrm{M})$ for $24 \mathrm{~h}$. (F) qRT-PCR analysis of the expression of TNF- $\alpha$, IL-I2, TGF- $\beta$ I, and IL-I0 in THP-I MO macrophages incubated with MVC and/or a STAT3 inhibitor (HJC0I52). (G) Representative Western blots illustrating the expression of the SOCS3 protein as well as total protein levels and phosphorylated-protein levels of JAK2, STAT3, p38 MAPK, ERKI/2, and cEBP/ $\beta$ in THP-I M0 macrophages cocultured with either HCCLM3 or Huh7 hepatoma cells subjected or not subjected to $8 \mathrm{~Gy}$ X-ray irradiation. The data are presented as mean \pm standard deviation from three independent experiments. $* P<0.05 ; * * P<0.01 ; * * * P<0.001 ; * * * * P<0.0001$.

Abbreviations: NC, negative control; MVC, maraviroc.

CCL5. After combined treatment with MVC and a STAT3 inhibitor (HJC0152), the phenotype of THP1 M0 macrophages returned to the M2 state (high IL10 and low IL-12 expression; Figure 3F). Similarly, in the coculture model of macrophages with HCCLM3 or Huh7 hepatoma cells, we further confirmed the role of the STAT3-SOCS3 pathway in the alteration of macrophage plasticity (Figure 3G).

\section{MVC Improves Radiation Sensitivity of Hepatoma Cells by Modulating Macrophage Plasticity}

To investigate whether the modulation of macrophage plasticity can influence the sensitivity of hepatoma cells to X-ray irradiation, a clonogenic radiosensitivity assay was conducted. First, THP-1 M0 macrophages were differentiated 
into M1 and M2 macrophages (Figure S2) and cocultured with either the HCCLM3 or Huh7 tumor cell line for 24 h. Next, the hepatoma cells were exposed to different doses of X-ray irradiation $(0,2,4,6$, or $8 \mathrm{~Gy})$ and cultured for 2 weeks. As shown in Figure 4A, both HCCLM3 and Huh7 hepatoma cells cocultured with M1-polarized macrophages had higher sensitivity to X-rays, whereas hepatoma cells cocultured with M2-polarized macrophages were significantly resistant to the irradiation as compared to the control tumor cells (not cocultured with macrophages) $(P<0.05)$. Given that MVC can skew macrophages toward the M1 phenotype, we incubated M2-polarized macrophages with MVC for $24 \mathrm{~h}$. After that, HCCLM3 and Huh7 hepatoma cells cocultured with the pretreated macrophages were subjected to the clonogenic radiosensitivity assay. The results revealed that the sensitivity of HCCLM3 and Huh7 cells to $\mathrm{X}$-ray irradiation statistically significantly increased (Figure
4B), accompanied by greater tumor cell apoptosis as evidenced by FCM (Figure 4C and D) and Western blotting data (Figure 4E).

\section{X-Ray Irradiation Combined with MVC Suppresses the Growth of Liver Tumor Cells in the Subcutaneous Tumor Model}

Given the in vitro results showing that MVC can indirectly raise the sensitivity of hepatoma cells to radiation by targeting the macrophage phenotype, we constructed a mouse model of a subcutaneous tumor (Figure 5A and B) to examine the combined effects of irradiation and MVC on subcutaneous growth of liver tumor cells. The mice were subdivided randomly into four groups: a negative control group, MVC administration group, irradiation group, and irradiation combined with MVC group. As presented in Figure 5C - E, MVC significantly suppressed tumor growth relative to the control group, whereas X-ray irradiation had a more favorable antitumor
A

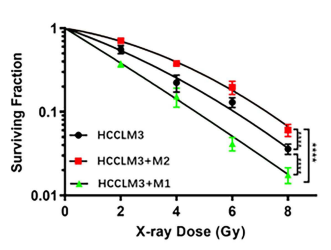

B

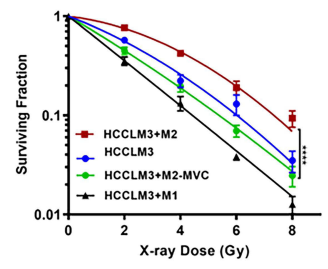

D

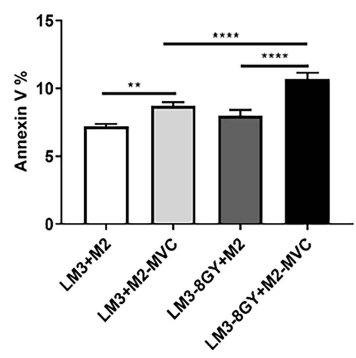

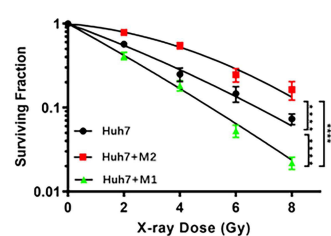

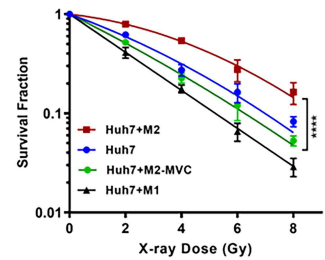

C

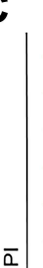

$\overline{\mathbf{n}}$

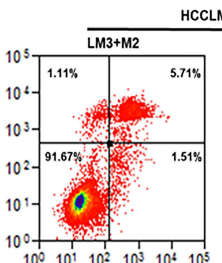

\begin{tabular}{lllll}
$10^{0}$ & $10^{1}$ & $10^{2}$ & $10^{3}$ & $10^{4}$ \\
$10^{5} \quad$ LM3-8GY+M2 & $10^{5}$ \\
\hline
\end{tabular}
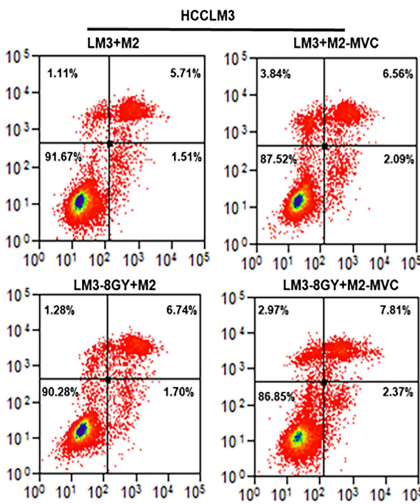

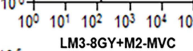
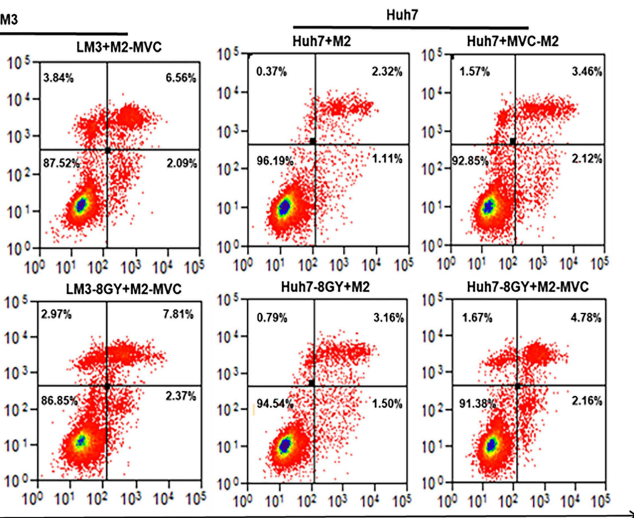

Annexin V (FITC)

$\mathbf{E}$

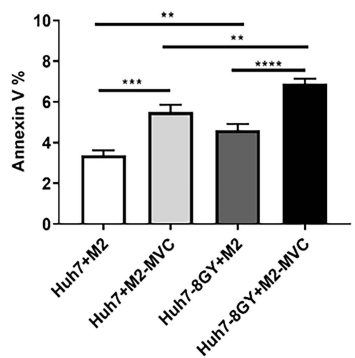

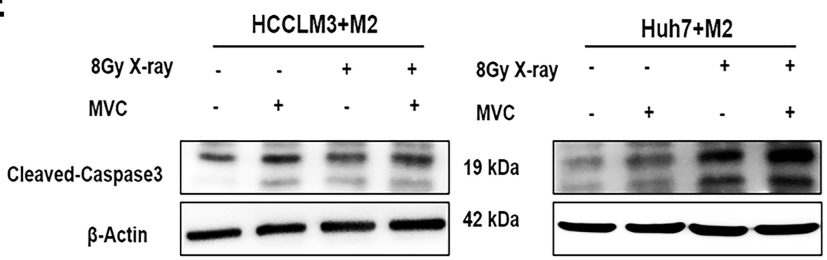

Figure 4 MVC improves the sensitivity of hepatoma cells to radiation by altering the macrophage phenotype. (A) Results of the clonogenic radiosensitivity assay indicating that HCCLM3 (left) and Huh7 (right) hepatoma cells cocultured with MI-polarized macrophages (green dots) have higher sensitivity to X-ray radiation, whereas hepatoma cells cocultured with M2-type macrophages (red dots) manifest radioresistance as compared with control cells (black dots). (B) HCCLM3 cells (left) or Huh7 cells (right) were cocultured with M2-polarized macrophages (subjected or not subjected to pretreatment with MVC for $24 \mathrm{~h}$ ) and plated for the clonogenic radiosensitivity assay. $P<$ 0.05 for both hepatoma cells cocultured with M2-phenotype macrophages pretreated with MVC when compared with hepatoma cells cocultured with macrophages not subjected to MVC pretreatment at all doses. (C and D) Apoptosis rates of HCCLM3 (left) and Huh7 (right) hepatoma cells cocultured with M2-polarized macrophages subjected or not subjected to pretreatment with MVC for $24 \mathrm{~h}$, as determined by flow cytometry. (E) Representative Western blots showing the expression of cleaved caspase 3 (an indicator of apoptosis) in hepatoma cells cocultured with M2-like macrophages subjected or not subjected to pretreatment with MVC. Values represent mean \pm standard deviation from three independent experiments. $* * P<0.01$ I $* * * P<0.001$; **** $P<0.000 \mathrm{I}$

Abbreviation: MVC, maraviroc. 
A

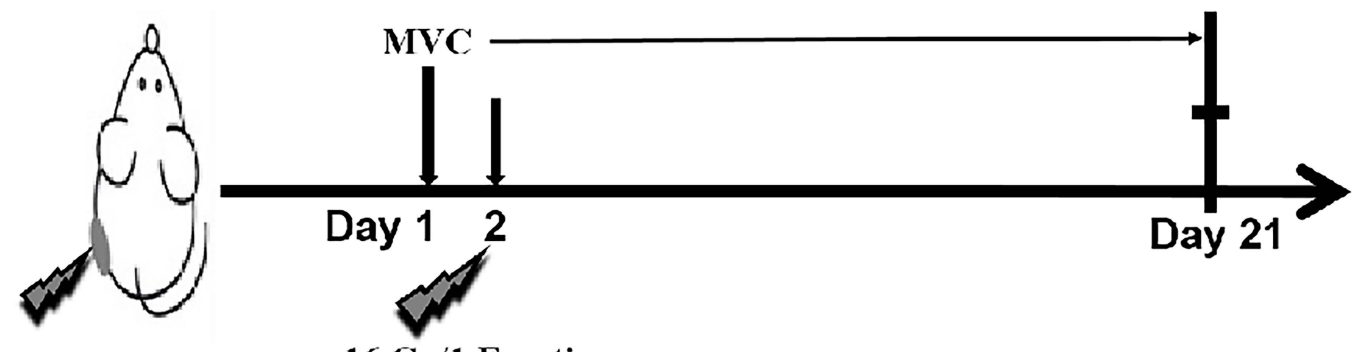

16 Gy/1 Fraction

B

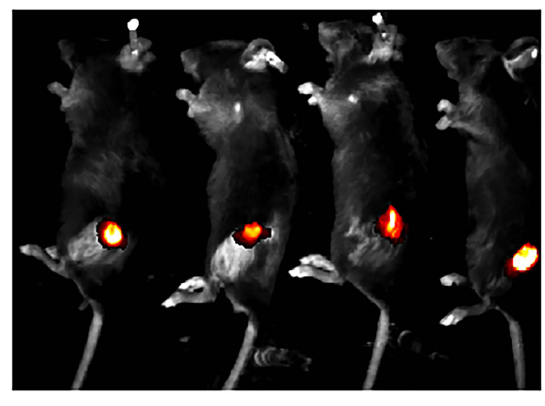

D

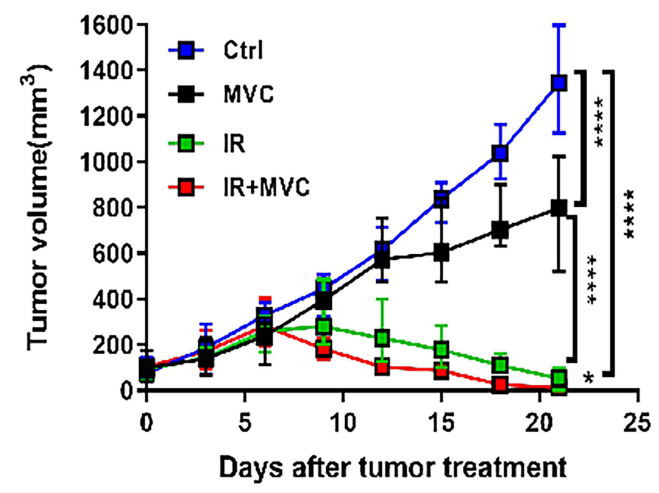

$\mathbf{F}$

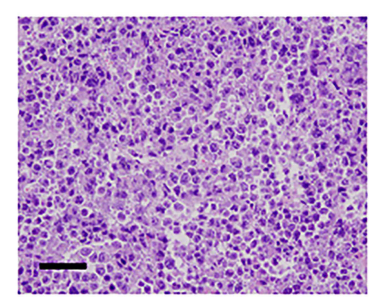

Ctrl

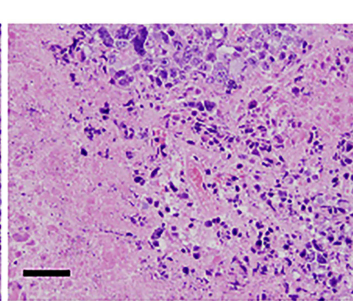

IR

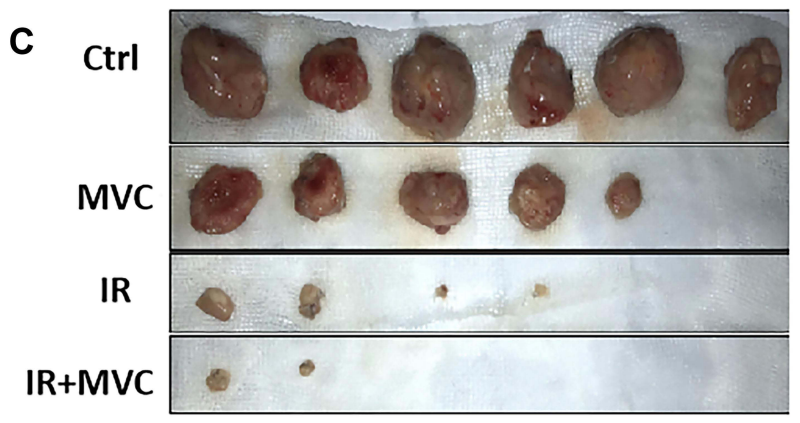

E

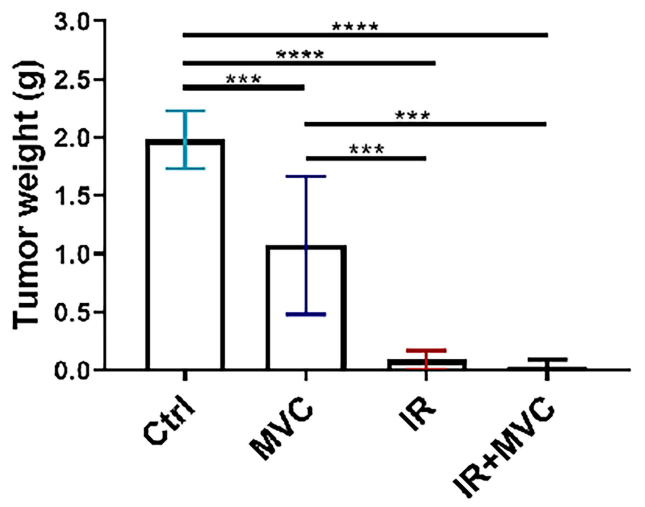

Figure 5 X-ray irradiation in combination with MVC suppress the growth of liver tumor cells in the mouse model of a subcutaneous tumor. C57BL/6) mice were randomly subdivided into four groups ( $\mathrm{n}=6$ for each group): a negative control group (labeled as Ctrl), MVC administration group (designated as MVC), $\mathrm{X}$-ray irradiation group (labeled as IR), and irradiation combined with MVC group (named IR+MVC). (A) MVC was injected intraperitoneally for 3 consecutive weeks. A dose of I6 Gy X-ray irradiation in I fraction with an energy of $6 \mathrm{MV}$ was applied to subcutaneous tumors. (B) In vivo imaging confirming that the C57BL/6) mouse model of a subcutaneous tumor was constructed successfully by means of luciferase-tagged $\mathrm{H} 22$ hepatoma cells. (C) After 3 weeks of treatments, the mice were killed by cervical dislocation, and the tumor tissues were excised. (D) The subcutaneous growth of liver tumor cells in C57BL/6 mice. Tumor growth in both X-ray irradiation and MVC groups was significantly slower as compared with the control group. The irradiation had a more favorable effect when compared with the control and the MVC group. The combined treatment with irradiation and MVC was more effective than either radiation or MVC alone. (E) The average subcutaneous tumor weight of each group at the time of sacrifice. (F) Representative hematoxylin-eosin images of tumor tissues from C57BL/6] mice (scale bar: $50 \mu \mathrm{m}$ ). Lower tumor cell density was observed in the three treatment groups compared with the control group. $* P<0.05 ; * * * P<0.001$; $* * * * P<0.0001$.

Abbreviations: Ctrl, control; IR, irradiation; MVC, maraviroc. 
effect as compared to the control and MVC groups $(P<$ 0.05). Notably, the combination of MVC and irradiation yielded the best treatment outcome. Consistent with these results, hematoxylin-eosin staining of tissue sections of subcutaneous tumors from the mice uncovered lower tumor cell density in the three treatment groups compared with the control group (Figure 5F). In summary, our results provide a theoretical basis for the combination of radiation therapy with immunotherapy targeting receptor CCR5 in HCC.

\section{Discussion}

Cancer can "hijack" chemokine networks to support cancer progression. Substantial evidence points to major participation of CCL 5 in the progression of liver diseases, especially HCC. $^{23,24,35}$ Singh et al have observed a higher expression level of CCL5 in HCC tissues than in non-neoplastic liver tissues and found that CCR5-CCL5 interaction causes metastatic behavior of HCC cells. ${ }^{24}$ Sadeghi et al have demonstrated that a higher serum level of CCL5 in patients with cirrhosis indicates the presence of HCC. ${ }^{35}$ Mohs et al have reported that CCL5 deficiency in bone marrowderived immune cells improves the prognosis of mice with liver cancer. ${ }^{23}$ Increasing evidence proves that CCL5 can promote tumor growth and metastasis by recruiting heterogeneous immune cells into a tumor site and by inducing the polarization of macrophages toward protumoral M2 subtypes. ${ }^{21,22,33,36,37}$ For example, Mi et al have found that THP-1 macrophages incubated with CCL5 exhibit upregulated expression of M2-related genes. ${ }^{22}$ Nieto et al have demonstrated that CCL5 is responsible for the upregulation of CD163 (a marker of M2 macrophages) in human monocytes. ${ }^{37}$ In line with these observations, the present study revealed that CCL5 $(5 \mathrm{ng} / \mathrm{mL})$ can promote the polarization of THP-1 macrophages toward the M2 phenotype. However, Li et al have reported that CCL5 $(100 \mathrm{ng} / \mathrm{mL})$ directly promotes M1 polarization of peritoneal macrophages and bone marrow-derived macrophages in mice. ${ }^{38}$ This discrepancy may be attributed to the different sources of macrophages or different concentrations of CCL5.

The current study supports the theory that CCL5 regulates macrophage functions by binding to CCR5. We proved that MVC - an FDA-approved well-tolerated CCR5 inhibitor with high specificity - can revert macrophage status to the antitumoral M1 phenotype. Furthermore, to simulate the relevant microenvironment in vivo, we established a coculture model of hepatoma cells and THP-1 macrophages. Evidence suggests that tumors receiving radiation therapy usually cause greater recruitment and influx of TAMs, ${ }^{39}$ while macrophages recruited to the tumor site are derived mainly from circulating blood-borne precursors such as monocytes. ${ }^{6,40}$ Therefore, most macrophages at a liver tumor site have not been exposed to X-rays, and consequently, only hepatoma cells in vitro were subjected to irradiation in our study. Notably, coculture with irradiated hepatoma cells significantly upregulated the markers of M2-polarized macrophages, such as IL-10 and TGF- $\beta$; this effect was markedly attenuated by MVC treatment. All of the above findings offer a new way to repolarize protumoral TAMs into antitumoral M1 macrophages by means of a CCR5 antagonist to inhibit HCC progression via blockage of CCL5-CCR5 signal transduction.

To gain a deeper insight into the mechanism of action of the CCL5-CCR5 axis in the regulation of the macrophage phenotype, Western blotting was conducted to evaluate the main pathways associated with CCL5-CCR5 signal transduction. ${ }^{26}$ The results indicated that in macrophages treated with CCL5, increased levels of SOCS3 were abrogated by the blockage of the CCL5-CCR5 axis with MVC, thereby leading to increased STAT3 levels typically linked to an M1polarized state. SOCS3 is a feedback inhibitor of the JAKSTAT3 pathway. Nonetheless, at present, there is still a controversy regarding the function of SOCS3 in the reprogramming of macrophage plasticity. Gordon et al have reported that silencing of SOCS3 upregulates the expression of M2 macrophage markers CD206 and transglutaminase, ${ }^{41}$ whereas Qin has proved that SOCS3 deficiency, which is characterized by a high STAT3 level, promotes M1 macrophage polarization, ${ }^{42}$ consistently with our data. In addition, studies on the effects of MVC on liver metastasis and breast cancer have confirmed our findings. ${ }^{21,33}$ The discrepancy in the function of SOCS3 in macrophage polarization may be attributed to the different sources of macrophages or differences in the treatments applied to tumors. We also found that when MVC and a STAT3 inhibitor were used together, the phenotype of macrophages repolarized to the M2 type. These observations are consistent with studies showing that JAK2STAT3 pathway inhibition can promote M2 macrophage polarization. ${ }^{43,44}$

Furthermore, our study revealed that the modulatory impact of MVC on macrophage polarization can in turn increase radiation sensitivity of hepatoma cells and promote tumor cell apoptosis. On the one hand, M2 macrophages can directly promote tumor cell growth by secreting cytokines such as IL-10. ${ }^{45}$ On the other hand, M2 macrophages can reduce the production of nitric oxide by blocking the 
inducible nitric oxide synthase pathway, thereby inhibiting tumor cell apoptosis. ${ }^{46,47}$ Additionally, studies have proved that IL-10 in the culture supernatant of M2 macrophages induces the expression of the tumor cell antiapoptotic gene B lymphocyte tumor 2, leading to drug resistance of tumor cells. ${ }^{48}$ In our study, macrophages receiving MVC tended to have M1-like properties with a decreased level of IL-10 in the supernatant. This phenomenon may partially explain the apoptosis of hepatoma cells and the increased sensitivity of tumors to radiation. In agreement with our results, Rahal et al have demonstrated that breast cancer cells cocultured with M1 macrophages have higher radiation sensitivity, whereas breast cancer cells cocultured with M2 macrophages manifest radioresistance. ${ }^{49}$

To date, MVC has shown its favorable antitumor performance in vitro and in animal models of breast, ${ }^{21,30}$ lung, ${ }^{31}$ and colorectal ${ }^{32,33}$ cancer; glioblastoma multiforme; ${ }^{50}$ and HCC. ${ }^{51}$ As for HCC, Barashi et al have found that Mdr2-and-CCR5 double-knockout mice exhibit a significant decrease in macrophage trafficking to the liver and in tumor incidence and size. ${ }^{25}$ Therefore, those authors proposed that the CCR5 antagonist MVC can be utilized for HCC prevention and treatment. Ochoa-Callejero et al have used MVC in a mouse model of diet-induced HCC to investigate whether this intervention can reduce disease progression. ${ }^{51}$ They found that animals treated with MVC show longer survival and a lower liver tumor burden than do their counterparts (controls) subjected to a hepatotoxic diet alone. Moreover, in a functional in vitro organ model of liver metastasis, MVC skews the polarization of TAMs toward the M1 phenotype thus activating an antitumor effect. ${ }^{33}$ This function was next confirmed in a Phase I trial involving patients with liver metastases from advanced colorectal cancer. ${ }^{33}$ Nevertheless, the effects of MVC in combination with radiation remain poorly understood. To investigate the efficacy of MVC in improving the antitumor effects of radiation, a mouse model of a subcutaneous tumor was constructed here by means of liver tumor cells. We observed that MVC combined with X-ray irradiation markedly suppressed tumor growth compared to the MVC-alone group or irradiation-alone group. Nevertheless, the key mechanisms behind their cooperative effects have not been clarified and deserve further investigation.

\section{Conclusion}

Blocking CCL5-CCR5 signal transduction by the CCR5 antagonist MVC induces a phenotypic shift in macrophages, which is mediated by STAT3-SOCS3 signaling and is referred to as a switch from the M2 to M1 phenotype.
Besides, macrophage repolarization raises the sensitivity of hepatoma cells to radiation. Targeting the CCL5-CCR5 axis is a promising approach to $\mathrm{HCC}$ treatment. In our future research, we will investigate the mechanism underlying the radiosensitivity of HCC cells to gain theoretical insight into the combination of radiation therapy with immunotherapy.

\section{Abbreviations}

HCC, hepatocellular carcinoma; TAM, tumor-associated macrophage; CCL, C-C chemokine ligand; CCR, C-C chemokine receptor; MVC, maraviroc; STAT, signal transducer and activator of transcription; SOCS, suppressor of cytokine signaling; RPMI, Roswell Park Memorial Institute; PMA, phorbol 12-myristate 13-acetate; IL, interleukin; TNF- $\alpha$, tumor necrosis factor- $\alpha$; TGF- $\beta$, transforming growth factor- $\beta$; mRNA, messenger RNA; siRNA, small interfering RNA; ARG-1, arginase-1; FCM, flow cytometry.

\section{Author Contributions}

All authors made substantial contributions to conception and design, acquisition of data, or analysis and interpretation of data; took part in drafting the article or revising it critically for important intellectual content; agreed to submit to the current journal; gave final approval for the version to be published; and agreed to be accountable for all aspects of the work.

\section{Funding}

The research was supported by a grant from the National Natural Science Foundation of China (No. 8160110123) and the National Key R\&D Program of China (2017YFC0112100).

\section{Disclosure}

The authors declare no competing interests inlcuding financial interests.

\section{References}

1. Zeng Z, Seong J, Yoon SM, et al. Consensus on stereotactic body radiation therapy for small-sized hepatocellular carcinoma at the 7 th Asia-Pacific primary liver cancer expert meeting. Liver Cancer. 2017;6:264-274. doi:10.1159/000475768

2. Chung YL, Jian JJ-M, Cheng SH. Sublethal irradiation induces vascular endothelial growth factor and promotes growth of hepatoma cells: implications for radiotherapy of hepatocellular carcinoma. Clin Cancer Res. 2006;12:2706-2715. doi:10.1158/1078-0432.CCR-05-2721

3. Zhou L, Wang Z, Gao Y, Wang L, Zeng Z. Stimulation of hepatoma cell invasiveness and metastatic potential by proteins secreted from irradiated nonparenchymal cells. Int J Radiation Oncol. 2012;84:822. doi:10.1016/j.ijrobp.2012.01.011 
4. Barker HE, Paget JTE, Khan AA, Harrington KJ. The tumour microenvironment after radiotherapy: mechanisms of resistance and recurrence. Nat Rev Cancer. 2015;15:409-425. doi:10.1038/nrc3958

5. Liu Y, Cao X. The origin and function of tumor-associated macrophages. Cell Mol Immunol. 2015;12:1-4. doi:10.1038/ cmi.2014.83

6. Mantovani A, Allavena P, Sica A. Tumour-associated macrophages as a prototypic type II polarised phagocyte population: role in tumour progression. Eur $J$ Cancer. 2004;40:1660-1667. doi:10.1016/j. ejca.2004.03.016

7. Chung Y, Yu C, Chiu S, et al. Diffusion-weighted MRI and 18F-FDG PET correlation with immunity in early radiotherapy response in BNL hepatocellular carcinoma mouse model: timeline validation. Eur J Nucl Med Mol I. 2019;46:1733-1744. doi:10.1007/s00259019-04318-3

8. Xu J, Escamilla J, Mok S, et al. CSF1R signaling blockade stanches tumor-infiltrating myeloid cells and improves the efficacy of radiotherapy in prostate cancer. Cancer Res. 2013;73:2782-2794. doi:10.1158/0008-5472.CAN-12-3981

9. Vatner RE, Formenti SC. Myeloid-derived cells in tumors: effects of radiation. Semin Radiat Oncol. 2015;25:18-27. doi:10.1016/j. semradonc.2014.07.008

10. Komohara Y, Jinushi M, Takeya M. Clinical significance of macrophage heterogeneity in human malignant tumors. Cancer Sci. 2014;105:1-8. doi:10.1111/cas.12314

11. Zhu X, Zhang J, Zhuang P, et al. High expression of macrophage colony-stimulating factor in peritumoral liver tissue is associated with poor survival after curative resection of hepatocellular carcinoma. J Clin Oncol. 2008;26:2707-2716. doi:10.1200/JCO.2007.15.6521

12. Yeung OWH, Lo $\mathrm{C}$, Ling $\mathrm{C}$, et al. Alternatively activated (M2) macrophages promote tumour growth and invasiveness in hepatocellular carcinoma. $J$ Hepatol. 2015;62:607-616. doi:10.1016/j. jhep.2014.10.029

13. Denardo DG, Ruffell B. Macrophages as regulators of tumour immunity and immunotherapy. Nat Rev Immunol. 2019;19:369-382. doi:10.1038/s41577-019-0127-6

14. Ngambenjawong C, Gustafson HH, Pun SH. Progress in tumor-associated macrophage (TAM)-targeted therapeutics. $A d v$ Drug Deliver Rev. 2017;114:206-221. doi:10.1016/j. addr.2017.04.010

15. Li X, Yao W, Yuan Y, et al. Targeting of tumour-infiltrating macrophages via CCL2/CCR2 signalling as a therapeutic strategy against hepatocellular carcinoma. Gut. 2016;66:157-167. doi:10.1136/gutjnl2015-310514

16. Liang C. Chemokines and their receptors play important roles in the development of hepatocellular carcinoma. World $J$ Hepatol. 2015;7:1390. doi:10.4254/wjh.v7.i10.1390

17. Marques RE, Guabiraba R, Russo RC, Teixeira MM. Targeting CCL5 in inflammation. Expert Opin Ther Targets. 2013;17:1439-1460. doi:10.1517/14728222.2013.837886

18. Walens A, Dimarco AV, Lupo R, Kroger BR, Damrauer JS, Alvarez JV. CCL5 promotes breast cancer recurrence through macrophage recruitment in residual tumors. Elife. 2019;8. doi:10.7554/ eLife.43653.

19. Oppermann M. Chemokine receptor CCR5: insights into structure, function, and regulation. Cell Signal. 2004;16:1201-1210. doi:10.1016/j.cellsig.2004.04.007

20. Onuffer JJ, Horuk R. Chemokines, chemokine receptors and small-molecule antagonists: recent developments. Trends Pharmacol Sci. 2002;23:459-467. doi:10.1016/s0165-6147(02)02064-3

21. Nie Y, Huang H, Guo M, et al. Breast phyllodes tumors recruit and repolarize tumor-associated macrophages via secreting CCL5 to promote malignant progression, which can be inhibited by CCR5 inhibition therapy. Clin Cancer Res. 2019;25:3873-3886. doi:10.1158/ 1078-0432.CCR-18-3421
22. Mi S, Qu Y, Chen X, Wen Z, Chen P, Cheng Y. Radiotherapy increases 12-LOX and CCL5 levels in esophageal cancer cells and promotes cancer metastasis via THP-1-derived macrophages. Onco Targets Ther. 2020;13:7719-7733. doi:10.2147/OTT.S257852

23. Mohs A, Kuttkat N, Reißing J, et al. Functional role of CCL5/ RANTES for HCC progression during chronic liver disease. J Hepatol. 2017;66:743-753. doi:10.1016/j.jhep.2016.12.011

24. Singh SK, Mishra MK, Rivers BM, Gordetsky JB, Bae S, Singh R. Biological and clinical significance of the CCR5/CCL5 axis in hepatocellular carcinoma. Cancers. 2020;12:883. doi:10.3390/ cancers 12040883

25. Barashi N, Weiss ID, Wald O, et al. Inflammation-induced hepatocellular carcinoma is dependent on CCR5 in mice. Hepatology. 2013;58:1021-1030. doi:10.1002/hep.26403

26. Aldinucci D, Colombatti A. The inflammatory chemokine CCL5 and cancer progression. Mediat Inflamm. 2014;2014:1-12. doi:10.1155/ 2014/292376

27. Gallo M, De Luca A, Lamura L, Normanno N. Zoledronic acid blocks the interaction between mesenchymal stem cells and breast cancer cells: implications for adjuvant therapy of breast cancer. Ann Oncol. 2012;23:597-604. doi:10.1093/annonc/mdr159

28. Borghese C, Cattaruzza L, Pivetta E, et al. Gefitinib inhibits the cross-talk between mesenchymal stem cells and prostate cancer cells leading to tumor cell proliferation and inhibition of docetaxel activity. J Cell Biochem. 2013;114:1135-1144. doi:10.1002/ jcb. 24456

29. Casagrande N, Borghese C, Visser L, Mongiat M, Colombatti A, Aldinucci D. CCR5 antagonism by maraviroc inhibits Hodgkin lymphoma microenvironment interactions and xenograft growth. Haematologica. 2019;104:564-575. doi:10.3324/haematol. 2018.196725

30. Pervaiz A, Zepp M, Mahmood S, Ali DM, Berger MR, Adwan H. CCR5 blockage by maraviroc: a potential therapeutic option for metastatic breast cancer. Cell Oncol. 2019;42:93-106. doi:10.1007/ s13402-018-0415-3

31. Halvorsen EC, Hamilton MJ, Young A, et al. Maraviroc decreases CCL8-mediated migration of CCR5+ regulatory T cells and reduces metastatic tumor growth in the lungs. Oncoimmunology. 2016;5: e1150398. doi:10.1080/2162402X.2016.1150398

32. Pervaiz A, Ansari S, Berger MR, Adwan H. CCR5 blockage by maraviroc induces cytotoxic and apoptotic effects in colorectal cancer cells. Med Oncol. 2015;32. doi:10.1007/s12032-015-0607-x.

33. Halama N, Zoernig I, Berthel A, et al. Tumoral immune cell exploitation in colorectal cancer metastases can be targeted effectively by anti-CCR5 therapy in cancer patients. Cancer Cell. 2016;29:587-601. doi:10.1016/j.ccell.2016.03.005

34. Dorr P, Westby M, Dobbs S, et al. Maraviroc (UK-427,857), a potent, orally bioavailable, and selective small-molecule inhibitor of chemokine receptor CCR5 with broad-spectrum anti-human immunodeficiency virus type 1 activity. Antimicrob Agents Ch. 2005;49:4721-4732. doi:10.1128/AAC.49.11.4721-4732.2005

35. Sadeghi M, Lahdou I, Oweira H, et al. Serum levels of chemokines CCL4 and CCL5 in cirrhotic patients indicate the presence of hepatocellular carcinoma. Br J Cancer. 2015;113:756-762. doi:10.1038/ bjc. 2015.227

36. Ban Y, Mai J, Li X, et al. Targeting autocrine CCL5-CCR5 axis reprograms immunosuppressive myeloid cells and reinvigorates antitumor immunity. Cancer Res. 2017;77:2857-2868. doi:10.1158/ 0008-5472.CAN-16-2913

37. Nieto JC, Zamora C, Cantó E, et al. CSF-1 regulates the function of monocytes in Crohn's disease patients in remission. Sci Rep. 2017;7:92. doi:10.1038/s41598-017-00145-4

38. Li M, Sun X, Zhao J, et al. CCL5 deficiency promotes liver repair by improving inflammation resolution and liver regeneration through M2 macrophage polarization. Cell Mol Immunol. 2020;17:753-764. doi:10.1038/s41423-019-0279-0 
39. Chew V, Lee YH, Pan L, et al. Immune activation underlies a sustained clinical response to Yttrium-90 radioembolisation in hepatocellular carcinoma. Gut. 2019;68:335-346. doi:10.1136/ gutjnl-2017-315485

40. Movahedi K, Laoui D, Gysemans C, et al. Different tumor microenvironments contain functionally distinct subsets of macrophages derived from Ly6C(high) monocytes. Cancer Res. 2010;70:5728-5739. doi:10.1158/0008-5472.CAN-09-4672

41. Gordon P, Okai B, Hoare JI, Erwig LP, Wilson HM. SOCS3 is a modulator of human macrophage phagocytosis. J Leukocyte Biol. 2016;100:771-780. doi:10.1189/jlb.3A1215-554RR

42. Qin H, Holdbrooks AT, Liu Y, Reynolds SL, Yanagisawa LL, Benveniste EN. SOCS3 deficiency promotes M1 macrophage polarization and inflammation. $J$ Immunol. 2012;189:3439-3448. doi:10.4049/jimmunol.1201168

43. Oh H, Park SH, Kang MK, et al. Asaronic acid attenuates macrophage activation toward M1 phenotype through inhibition of NF-kappaB pathway and JAK-STAT signaling in glucose-loaded murine macrophages. J Agric Food Chem. 2019;67:10069-10078. doi:10.1021/acs.jafc.9b03926

44. Wang S, Cao M, Xu S, et al. Luteolin alters macrophage polarization to inhibit inflammation. Inflammation. 2020;43:95-108. doi:10.1007/ s10753-019-01099-7

45. Domínguez-Soto A, Sierra-Filardi E, Puig-Kröger A, et al. Dendritic cell-specific ICAM-3-grabbing nonintegrin expression on M2polarized and tumor-associated macrophages is macrophage-CSF dependent and enhanced by tumor-derived IL-6 and IL-10. J Immunol. 2011;186:2192-2200. doi:10.4049/jimmunol.1000475
46. Nath N, Kashfi K. Tumor associated macrophages and 'NO'. Biochem Pharmacol. 2020;176:113899. doi:10.1016/j.bcp.2020. 113899

47. Chanmee T, Ontong P, Konno K, Itano N. Tumor-associated macrophages as major players in the tumor microenvironment. Cancers. 2014;6:1670-1690. doi:10.3390/cancers6031670

48. Yang $\mathrm{C}, \mathrm{He} \mathrm{L}, \mathrm{He} \mathrm{P}$, et al. Increased drug resistance in breast cancer by tumor-associated macrophages through IL-10/STAT3/bcl-2 signaling pathway. Med Oncol. 2015;32:352. doi:10.1007/s12032-0140352-6

49. Rahal OM, Wolfe AR, Mandal PK, et al. Blocking interleukin (IL)4and IL13-mediated phosphorylation of STAT6 (Tyr641) decreases M2 polarization of macrophages and protects against macrophage-mediated radioresistance of inflammatory breast cancer. Int $J$ Radiat Oncol. 2018;100:1034-1043. doi:10.1016/j. ijrobp.2017.11.043

50. Kranjc MK, Novak M, Pestell RG, Lah TT. Cytokine CCL5 and receptor CCR5 axis in glioblastoma multiforme. Radiol Oncol. 2019;53:397-406. doi:10.2478/raon-2019-0057

51. Ochoa-Callejero L, Pérez-Martínez L, Rubio-Mediavilla S, Oteo JA, Martínez A, Blanco JR. Maraviroc, a CCR5 antagonist, prevents development of hepatocellular carcinoma in a mouse model. PLoS One. 2013;8:e53992. doi:10.1371/journal.pone.0053992
Journal of Hepatocellular Carcinoma

\section{Publish your work in this journal}

The Journal of Hepatocellular Carcinoma is an international, peerreviewed, open access journal that offers a platform for the dissemination and study of clinical, translational and basic research findings in this rapidly developing field. Development in areas including, but not limited to, epidemiology, vaccination, hepatitis therapy, pathology and molecular tumor classification and prognostication are all considered for publication. The manuscript management system is completely online and includes a very quick and fair peer-review system, which is all easy to use. Visit http://www.dovepress.com/ testimonials.php to read real quotes from published authors. 